\title{
Some problems of non-associative combinations (2)
}

\author{
By A. Erdílyi and I. M. H. Etherington.
}

\$1. The preceding Note has shown the connection between the partition of a convex polygon by non-crossing diagonals and the insertion of brackets in a product, the latter being more commonly represented by the construction of a tree. It was shown that the enumeration of these entities leads to a generating function $y=f(x)$ which satisfies an algebraic equation of the type

$$
y=x+y^{a}+y^{b}+\ldots \quad(a, b, \ldots>1) .
$$

In simple cases, the solution of the equation was found as a power series in $x$, the coefficient $A_{n}$ of $x^{n}$ giving the required number of partitions of an $(n+1)$-gon.

Now it has been shown by several writers ${ }^{1}$ that every root of an algebraic equation can be expressed as a hypergeometric function of the coefficients of the equation. The generating function must thus be expressible as a hypergeometric function of $x$, from which an explicit formula for $A_{n}$ might be deduced. The formulae obtained by these writers, however, are not immediately applicable here, because the form of the equation generally assumed differs from (1). It is of course possible to transform (1) into the usual form; but then the application of the known formulae leads only to an expansion of $y$ in descending powers of $x$. Instead of converting this into a series of ascending powers as required for our problem, we have found it simpler to attack the equation afresh. As a matter of fact, our work is closely related to Birkeland's method, the device used being equivalent to his application of Lagrange's inversion formula ${ }^{2}$.

We shall show in $\S \S 2,3$ that the coefficient of $x^{n}$ in the generating function which is a solution of $(1)$ is

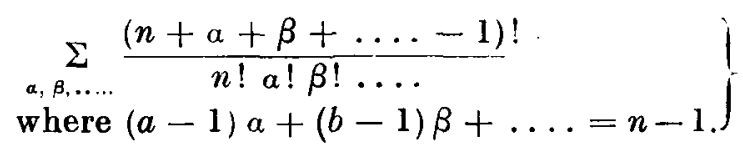

1 See e.g. Birkeland, several notes in Comptes rendus, 171 (1920) and $172(1921)$; Belardinelli, Annali di Mat. (3), 29 (1920), 251, Rend. de Lincei (5), 30) (1920), 208, Rend. di Palermo, 46 (1922), 463 ; Mayr, Monatshefte für Math. und Phys., 4õ (193i), 280 and 47 (1938), 164 ; Mellin, Ann. Acad. Sci. Fenn. (A) 7 (1914-15), Nos. 7 and 8.

${ }^{2}$ Cf. Whittaker and Watson, Modern Analysis (1927), $\$ 7.32$.

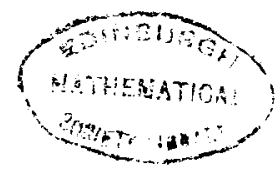


viii

This therefore represents the number of partitions of an $(n+1)$-gon into $(a+1)$-gons, $(b+1)$-gons, $\ldots$ Having obtained this result, it was natural to look for an interpretation of each term in this summation. This led us to consider a more specialised problem, that of enumerating the partitions of an $(a+1)$-gon into $a(a+1)$-gons, $\beta(b+1)$-gons, $\ldots \ldots$, where $n, a, a, \beta, b, \ldots$ are all given numbers. Beginning therefore with this problem we shall establish a more fundamental formula (8), from which (2) follows as an easy corollary.

As in the preceding Note, the solution depends in the first instance on the derivation of a non-linear recurrence relation. Now the earlier writers ${ }^{1}$ who considered the particular case of partition into triangles $(a=2, a=n-1, \beta=\ldots=0$; Case $I$ of the preceding Note) observed that the non-linear recurrence relation which occurs there is equivalent to the linear recurrence relation

$$
B_{n}=2(2 n-3) B_{n-1} \quad\left(B_{n}=n ! A_{n}\right),
$$

suitable initial conditions being prescribed in both cases. This connection between a non-linear and a linear recurrence equation persists in the problem just enunciated, but seemingly not in the less detailed problem with which we started. This will be shown in $\S 4$; the argument used is a generalisation of that of Rodrigues (loc. cit., p. 549).

$\S 2$. We consider for simplicity a special case $(a=2, b=3)$ of the problem enunciated in $\S 1$. Suppose that a convex $(n+1)$-gon is partitioned into $a$ triangles and $\beta$ quadrilaterals. It will appear from the solution that we must have

$$
n=\alpha+2 \beta+1 ;
$$

and this may be also shown directly without difficulty. Nevertheless it is convenient to regard the number of partitions as depending on $n, a, \beta$ independently; it will be denoted $A_{n a \beta}$, this being zero if the condition (3) is not satisfied. We define $A_{100}=1$.

Let one side $P Q$ of the polygon be selected as base. Suppose first that the part of the polygon which contains the base is a triangle $P Q R$; that $P R$ is the base of an $\left(n^{\prime}+1\right)$-gon divided into $a^{\prime}$ triangles and $\beta^{\prime}$ quadrilaterals; and that $Q R$ is the base of an $\left(n^{\prime \prime}+1\right)$-gon divided into $\alpha^{\prime \prime}$ triangles and $\beta^{\prime \prime}$ quadrilaterals. We must evidently have

$$
n^{\prime}+n^{\prime \prime}=n, \alpha^{\prime}+a^{\prime \prime}+1=\alpha, \beta^{\prime}+\beta^{\prime \prime}=\beta,
$$

I Lamé, Catalan, Rodrigues, Binet, Journ. de Math., 1838 39. 
the term " +1 " accounting for the triangle $P Q R$. The number of ways in which this can occur is $A_{n^{\prime} a^{\prime} \beta^{\prime}} A_{n^{\prime \prime} a^{\prime \prime} \beta^{\prime \prime}}$; and hence the total number of partitions of the original polygon in which $P Q$ forms one side of a triangle is

$$
\sum A_{n^{\prime} a^{\prime} \beta^{\prime}} A_{n^{\prime \prime} a^{\prime \prime} \beta^{\prime \prime}},
$$

the summation being over all values of the suffixes satisfying (4).

We can enumerate similarly the partitions which contain a quadrilateral $P Q R S$; and we arrive at the recurrence relation

$$
A_{n \alpha \beta}=\sum A_{n^{\prime} \alpha^{\prime} \beta^{\prime}} A_{n^{\prime \prime} a^{\prime \prime} \beta^{\prime \prime}}+\sum A_{n^{\prime} a^{\prime} \beta^{\prime}} A_{n^{\prime \prime} a^{\prime \prime} \beta^{\prime \prime}} A_{n^{\prime \prime \prime} \alpha^{\prime \prime \prime} \beta^{\prime \prime \prime}}(n>1) \text {, }
$$

where in the first summation

$$
n^{\prime}+n^{\prime \prime}=n, \quad \alpha^{\prime}+a^{\prime \prime}=\alpha-1, \quad \beta^{\prime}+\beta^{\prime \prime}=\beta,
$$

and in the second

$$
n^{\prime}+n^{\prime \prime}+n^{\prime \prime \prime}=n, \quad \alpha^{\prime}+\alpha^{\prime \prime}+\alpha^{\prime \prime \prime}=\alpha, \quad \beta^{\prime}+\beta^{\prime \prime}+\beta^{\prime \prime \prime}=\beta-1 .
$$

Introducing the generating function

$$
y=f(x, A, B)=\sum A_{n \alpha \beta} x^{n} A^{a} B^{\beta},
$$

it follows from (5) that

$$
y=x+A y^{2}+B y^{3} .
$$

By the same argument, it will be seen that the number $A_{n a \beta}$. of partitions of an $(n+1)$-gon into $a(a+1)$-gons, $\beta(b+1)$-gons, $\ldots$. is the coefficient of $x^{n} A^{a} B^{\beta} \ldots$ in the expansion of $y$, where $y$ is that root of the equation

$$
y=x+A y^{a}+B y^{b}+\ldots
$$

which vanishes for $x=0$.

§3. Instead of $(6)$, we consider the rather more general equation

$$
x=y F(y)
$$

where $F(y)$ is an analytic function of the complex variable $y$, onevalued and regular in a certain neighbourhood of $y=0$. We assume $F^{\prime}(0) \neq 0$, and may take without loss of generality $F(0)=1$ (otherwise replace $x$ by $x F(0)$ and divide the equation by $F(0))$. The solution of this equation presents no greater difficulties than that of (6).

Then there is one and only one branch of the function $y=f(x)$ which vanishes for $x=0$. This branch is an analytic function of $x$, one-valued and regular in a certain domain of the $x$-plane. We 
suppose $x$ (and later on $\xi$ ) to be inside this domain and $y$ (and $\eta$ ) to be inside the corresponding domain in the $y$-plane.

Now consider the integral

$$
2 \pi i \int_{C} \frac{d \eta}{\eta F(\eta)-x}
$$

in which the contour $C$ of the $\eta$-plane encircles $\eta=y$ in the positive direction remaining throughout inside the domain of one-to-one correspondence between $\eta$ and $\xi=\eta F(\eta)$. Introducing $\xi$ as a new variable of integration we obtain

$$
\frac{1}{2 \pi i} \int_{C^{\prime}} \frac{d \eta}{d \xi} d \xi /(\xi-x)
$$

$C^{\prime}$ is the image of $C$ in the $\xi$-plane and encircles $\xi=x$ in the positive direction. $\eta$ and hence $d \eta / d \xi$ being one-valued analytic functions, regular in a domain containing $C^{\prime}$ entirely, the value of the integral is

$$
\frac{d \eta}{d \xi}{ }^{\xi=x}=\frac{d y}{d x} .
$$

On the other hand, if $x$ is sufficiently small, we can choose a contour $C$ which is a circle with origin $\eta=0$, and in every point of which $|x|<|\eta F(\eta)|$. Then the expansion

$$
\frac{1}{\eta F(\eta)-x}=\frac{1}{\eta F(\eta)}\left(1-\frac{x}{\eta F(\eta)}\right)^{-1}=\sum_{n=2}^{\infty} \frac{x^{n-1}}{\{\eta F(\eta)\}^{n}}
$$

is uniformly convergent on $C$ and may be integrated term by term. Hence

$$
\frac{d y}{d x}=\frac{1}{2 \pi i} \int_{C} \frac{d \eta}{\eta F(\eta)-x}=\sum_{n=1}^{\infty} x^{n-1} \frac{1}{2 \pi i} \int^{(0+)} \frac{d \eta}{\eta^{n} F^{n}(\eta)}
$$

and, $f(0)$ being equal to zero,

$$
y=\int_{0}^{x} \frac{d y}{d x} d x=\sum_{n=1}^{\infty} \frac{x^{n}}{n} \frac{1}{2 \pi i} \int^{(0+)} \frac{d \eta}{\eta^{n} F^{n}(\eta)} .
$$

This result can be expressed as follows: the coefficient of $x^{n} / n$ in the expansion of $y$ in ascending powers of $x$ is equal to the coefficient of $y^{n-1}$ in the expansion of $\{F(y)\}^{-n}$ in ascending powers of $y$.

Let us apply this rule to the algebraic equation (6). In this case

$$
F(y)=1-A y^{a-1}-B y^{b-1}-\ldots \text {; }
$$

and hence by the multinomial theorem

$$
\{F(y)\}^{-n}=\sum_{a, \beta, \ldots} \frac{(n+\alpha+\beta+\ldots-1) !}{(n-1) ! \alpha ! \beta ! \ldots} A^{a} B^{\beta} \ldots y^{(a-1) a+(b-1) \beta+\ldots},
$$


the summation being extended over all non-negative integer values of $a, \beta, \ldots$ The coefficient of $y^{n-1}$ in this expansion is

$$
\underset{a, \beta, \ldots}{\sum}(n+\alpha+\beta+\ldots-1) ! A^{\alpha} B^{\beta} \ldots
$$

where $(a-1) a+(b-1) \beta+\ldots=n-1$.

Hence we get by our rule

$$
y=f(x)=\sum_{n=1}^{\infty} \frac{x^{n}}{n} \sum_{a, \beta, \ldots} \frac{(n+a+\beta+\ldots \ldots-1) !}{(n-1) ! \alpha ! \beta ! \ldots} A^{\alpha} B^{\beta} \ldots
$$

where $(a-1) a+(b-1) \beta+\ldots=n-1$ in the inner sum; that is, $y=\Sigma \frac{(a a+b \beta+\ldots) !}{[(a-1) \alpha+(b-1) \beta+\ldots+1] ! \alpha ! \beta ! \ldots} x^{(a-1) a+(b-1) \beta+\ldots A^{\alpha} B^{\beta}} \ldots$.
$(\alpha, \beta, \ldots=0,1,2, \ldots)$.

We have thus shown that

provided that

$$
A_{\text {na } \ldots .}=\left(n+\frac{a+\beta+\ldots-1) !}{n ! \alpha ! \beta ! \ldots}\right.
$$

$$
n=(a-1) a+(b-1) \beta+\ldots+1 ;
$$

and is otherwise zero.

Returning to the original problem where $a, \beta, \ldots$ are not specified, we have to deal with equation (1), and thus merely to put in (6)

$$
A=B=\ldots=1 \text {. }
$$

Correspondingly (2) follows from (7); or may be deduced from (8).

$\S 4$. Dropping the redundant suffix $n$ used in $\S 2$, let $A_{\alpha \beta}$ denote the number of partitions of a polygon with $\alpha+2 \beta+2$ sides into $\alpha$ triangles and $\beta$ quadrilaterals. This may also be interpreted as the number of associations of $\alpha+2 \beta+1$ identical elements in the form of a product containing $\alpha$ couplets and $\beta$ triplets. (A couplet or a triplet means a pair of brackets which unites two or three factors respectively.) Hence

$$
B_{a \beta}=(\alpha+2 \beta+1) ! A_{\alpha \beta}=\frac{(2 \alpha+3 \beta) !}{\alpha ! \beta !}
$$

enumerates the associations of $\alpha+2 \beta+1$ distinct elements in the same form. Iet us call these $B_{\alpha \beta}$ non-associative products the associations $(\alpha, \beta)$.

Suppose that a particular element in an association $(\alpha, \beta)$ is obliterated. The element may be one of a couplet or one of a triplet, and the association becomes accordingly either $(\alpha-1, \beta)$ or 
xii

$(\alpha+1, \beta-1)$. Conversely, any association $(\alpha-1, \beta)$ may be reconverted into $(\alpha, \beta)$ by the insertion of a single element in

$$
2\{1+2(\alpha-1)+3 \beta\}=2(2 \alpha+3 \beta-1)
$$

ways; for the element may be introduced as a pre- or post-multiplier of the whole association, of either factor in any of the $\alpha-1$ couplets or of one factor in any of the $\beta$ triplets. Similarly any association $(\alpha+1, \beta-1)$ may be reconverted into $(\alpha, \beta)$ by inserting a single element in $3(\alpha+1)$ different ways; for it may be introduced in threo positions into any of the $a+1$ couplets.

Consider now all the associations $(\alpha, \beta)$ of $\alpha+2 \beta+1$ given elements. When a particular element is obliterated, we are left with all the associations $(\alpha-1, \beta)$ each repeated $2(2 \alpha+3 \beta \ldots 1)$ times, and all the associations $(\alpha+1, \beta-1)$ each repeated $3(\alpha+1)$ times. Thus

$$
B_{a, \beta}=2(2 \alpha+3 \beta-1) B_{a-1, \beta}+3(\alpha+1) B_{a+1, \beta-1} .
$$

The argument may be generalised without difficulty. Taking in (8)

$$
a=2, b=3, \ldots
$$

and dropping the redundant suffix $n$, we obtain

$$
B_{a \beta \gamma \ldots .}=(\alpha+2 \beta+3 \gamma+\ldots+1) ! A_{a \beta \gamma \ldots}=\frac{(2 \alpha+3 \beta+4 \gamma+\ldots) !}{a ! \beta ! \gamma ! \ldots}
$$

as the number of associations of $a+2 \beta+3 \gamma+\ldots+1$ distinct elements in the form of a bracketed product containing $\alpha$ couplets, $\beta$ triplets, $\gamma$ quadruplets, ....; and it will be found that $\boldsymbol{B}_{a \beta \gamma} \ldots$ satisfies the linear recurrence relation

$$
\begin{aligned}
B_{a, \beta, \gamma} \ldots . & =2(2 \alpha+3 \beta+4 \gamma+\ldots-1) B_{a-1, \beta, \gamma, \ldots} \\
& +3(\alpha+1) B_{a+1, \beta-1, \gamma} \ldots+4(\beta+1) B_{a, \beta+1, \gamma-1, \ldots}+\ldots
\end{aligned}
$$

this formula being easily verified by direct substitution of (9).

The University, Edinbdrgh.

NOTE. Added 28th January, 1940.

Since this paper was written, a paper of G. Belardinelli (Monatsh. Math. Phys. 48 (1939), 381-388) has appeared which contains the general result of $\S 3$, namely the formula

$$
y=\sum_{n=1}^{\infty} \frac{x^{n}}{n} \cdot \frac{1}{2 \pi i} \int^{(0+)} \frac{d \eta}{\eta^{n} \frac{F^{n}(\eta)}{}}
$$

From this expression Belardinelli proves $y$ to be a hypergeometric series of infinite order in $x$. 